\title{
Small optic suspensions for Advanced LIGO input optics and other precision optical
}

experiments

G. Ciani, M. A. Arain, S. M. Aston, D. Feldbaum, P. Fulda, J. Gleason, M. Heintze, R. M. Martin, C. L.

Mueller, D. M. Nanda Kumar, A. Pele, D. H. Reitze, P. Sainathan, D. B. Tanner, L. F. Williams, and G. Mueller

Citation: Rev. Sci. Instrum. 87, 114504 (2016); doi: 10.1063/1.4967716

View online: http://dx.doi.org/10.1063/1.4967716

View Table of Contents: http://aip.scitation.org/toc/rsi/87/11

Published by the American Institute of Physics 


\title{
Small optic suspensions for Advanced LIGO input optics and other precision optical experiments
}

\author{
G. Ciani, ${ }^{1, a)}$ M. A. Arain, ${ }^{1, b)}$ S. M. Aston, ${ }^{2}$ D. Feldbaum, ${ }^{1}$ P. Fulda, ${ }^{1}$ J. Gleason, ${ }^{1}$ \\ M. Heintze, ${ }^{1}$ R. M. Martin, ${ }^{1}$ C. L. Mueller, ${ }^{1}$ D. M. Nanda Kumar, ${ }^{1}$ A. Pele,${ }^{2}$ D. H. Reitze,$\left.{ }^{1, c}\right)$ \\ P. Sainathan, ${ }^{1}$ D. B. Tanner, ${ }^{1}$ L. F. Williams,${ }^{1}$ and G. Mueller ${ }^{1}$ \\ ${ }^{1}$ University of Florida, Gainesville, Florida 32611, USA \\ ${ }^{2}$ LIGO Livingston Observatory, Livingston, Louisiana 70754, USA
}

(Received 29 June 2016; accepted 31 October 2016; published online 30 November 2016)

\begin{abstract}
We report on the design and performance of small optic suspensions developed to suppress seismic motion of out-of-cavity optics in the input optics subsystem of the Advanced Laser Interferometer Gravitational Wave Observatory. These compact single stage suspensions provide isolation in all six degrees of freedom of the optic, local sensing and actuation in three of them, and passive damping for the other three. Published by AIP Publishing. [http://dx.doi.org/10.1063/1.4967716]
\end{abstract}

\section{INTRODUCTION}

On September 14th, 2015, at the beginning of their first observing run, the Advanced Laser Interferometer Gravitational Wave Observatory (LIGO) made the first direct detection of gravitational waves. ${ }^{1}$ For about 4 months, although not yet at full sensitivity, the two instruments routinely operated with a range between 70 and $80 \mathrm{Mpc}$ for a reference NSNS binary system, observing a volume more than 50 times larger than their predecessors. ${ }^{2}$ Critical to this success has been the performance of the Input Optics (IO) subsystem, ${ }^{3}$ designed, built, installed, and tested by the LIGO group at the University of Florida; the subsystem is charged with delivering a stable and well-shaped beam to the main interferometer across the whole range of possible operating input powers, up to $180 \mathrm{~W}$. The in-vacuum portion of the IO subsystem employs $75 \mathrm{~mm}$ diameter optics to steer and mode-match the laser beam from the input mode cleaner (IMC) into the power recycled interferometer; these out-of-cavity optics are suspended by small, single stage vacuum compatible suspensions called HAM auxiliary suspensions (HAUX) to isolate them from residual vibration of the optical table and to allow for pointing and local damping. A similar suspension system with different requirements, the HAM Tip-Tilt suspension (HTTS), is installed at the output chain of the LIGO detector. ${ }^{4}$ Both designs were derived from the small optic suspensions (SOS) employed in the input optic chain of initial LIGO. 5,6

Although developed in the context of Advanced LIGO, the HAUX can find application in a broader range of lab-scale optical experiments. They provide isolation in all degrees of freedom, local sensing and actuation, and active and passive damping, while employing a compact, lightweight mechanical design with a number of expedients to simplify operation and maintenance of the suspension and the installed optic.

\footnotetext{
a)Electronic mail: ciani@phys.ufl.edu

b) Current address: Carl Zeiss Meditec, Dublin, CA 94568, USA

c) Current address: LIGO Laboratory, California Institute of Technology,

Pasadena, CA 91125, USA.
}

This paper describes the requirements, design, and performance of the HAUX. Section II lists the performance requirements and desired operating characteristics and explains how they have driven the top level design choices. Section III describes the mechanical setup in greater detail, and the design expedients put in place to make assembly and maintenance of the suspension more convenient. Section IV presents data from the main performance tests performed on typical HAUX production units. Section V concludes this manuscript by summarizing the HAUX main strengths. In the Appendix we provide approximate formulas to easily estimate the resonant frequencies of the system based on few design parameters.

Detailed documentation regarding the requirements, final design, and testing of the HAUX can be accessed from the LIGO Document Control Center ${ }^{7}$ under entry number LIGO-E1600169. ${ }^{8}$

\section{REQUIREMENTS AND CONCEPTUAL DESIGN}

As shown in Fig. 1, the four optics suspended by the HAUX are all located on a single Advanced LIGO seismic isolation table, after the input mode cleaner (IMC) and before injection into the power-recycling cavity (PRC) of the main interferometer. The conceptual structure of the HAUX suspension chain has been based on the need of both precisely controlling the alignment of the input beam with respect to the main interferometer and preserving the noise performance of the input optic subsystem. In particular, the frequency and pointing noise introduced by the HAUX are generally required to be at least a factor 10 below the noise at the output of the IMC.

Noise in the displacement $x$ along the optical axis of a reflecting optic causes a variation of the total optical path and appears as frequency noise $S_{v}^{\frac{1}{2}}=\frac{4 \pi}{\lambda} f S_{x}^{\frac{1}{2}}$ in the beam downstream of the optic (where $S_{q}^{\frac{1}{2}}$ is used to indicate the amplitude spectral density of the quantity $q$ which has power spectral density equal to $S_{q}$ ). In addition, rotational noise of the optic around the horizontal or vertical axis orthogonal to the optical axis (referred to as pitch and yaw, respectively; see naming 


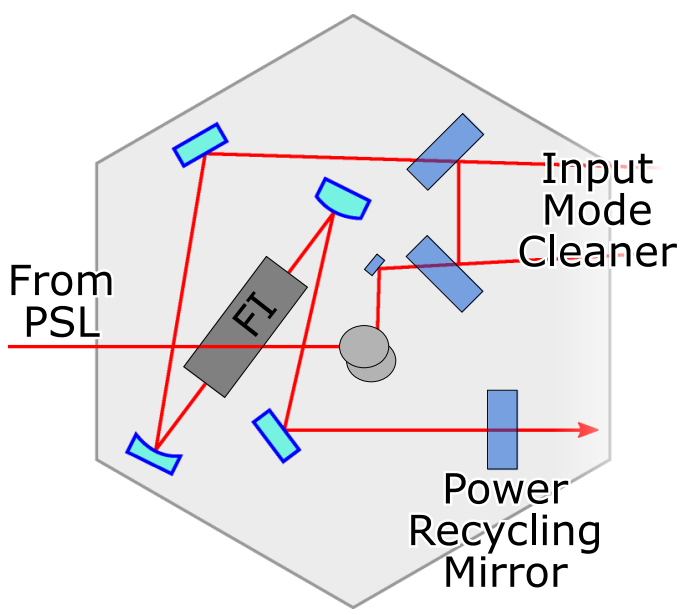

FIG. 1. A simplified representation of the HAM2 table which hosts the majority of the in-vacuum input optics. The laser beam coming from the prestabilized laser (PSL), on the left in the picture, propagates over the Faraday isolator (FI) and is lowered to table-height via the periscope in the center of the table. A small fixed optic steers it into the triangular input mode cleaner (IMC), of which only two optics are shown here. The mirrors installed in the four HAM auxiliary suspension, depicted in light blue on the left portion of the HAM2 table, route the beam, filtered and stabilized by the IMC, through the FI and towards the power recycling mirror. Two of these mirrors are curved and provide mode matching to the main interferometer.

of degrees of freedom in Fig. 2) directly couples into beam pointing noise so that $S_{\theta}^{\frac{1}{2}}=2 S_{\text {pitch,yaw }}^{\frac{1}{2}}$; this equation is strictly valid for a flat mirror and approximately valid for a curved mirror when the distance between the reflecting surface and the center of rotation (often assumed to be the center of mass of the physical mirror) is small compared to the radius of curvature.

Based on IMC requirements, ${ }^{9}$ assuming that the four mirrors experience uncorrelated noise and conservatively neglecting the effect of non-normal angle of incidence, for a single suspension this translates into a requirement on the residual displacement noise of

$$
\begin{aligned}
& S_{x}^{1 / 2} \leq 2 \cdot 10^{-11} \frac{\mathrm{m}}{\sqrt{\mathrm{Hz}}} \text { at } 10 \mathrm{~Hz}, \\
& S_{x}^{1 / 2} \leq 4 \cdot 10^{-14} \frac{\mathrm{m}}{\sqrt{\mathrm{Hz}}} \text { at } 100 \mathrm{~Hz},
\end{aligned}
$$

and on the residual rotational noise expressed as

$$
S_{\alpha}^{1 / 2} \leq 6 \cdot 10^{-13} \sqrt{1+\left(\frac{100 \mathrm{~Hz}}{f}\right)^{4}} \frac{\mathrm{rad}}{\sqrt{\mathrm{Hz}}} .
$$

These requirements are valid above $10 \mathrm{~Hz}$, which is the lower limit of the Advanced LIGO measurement band.

In general, one degree of freedom of a single stage mechanical suspension can be modeled (under ideal conditions) as a harmonic oscillator with a natural resonant frequency $f_{0}$. For frequencies $f$ above resonance and assuming negligible damping, the displacement response of the suspended mass to external forces quickly approaches that of a free mass, decaying as $1 /\left(m\left(f_{0}^{2}-f^{2}\right)\right)$. The response to motion of the suspension point shows a similar decay with frequency and goes as $f_{0}^{2} /\left(f_{0}^{2}-f^{2}\right)$. The level of isolation at a given frequency can thus be controlled by an appropriate choice of $f_{0}$.

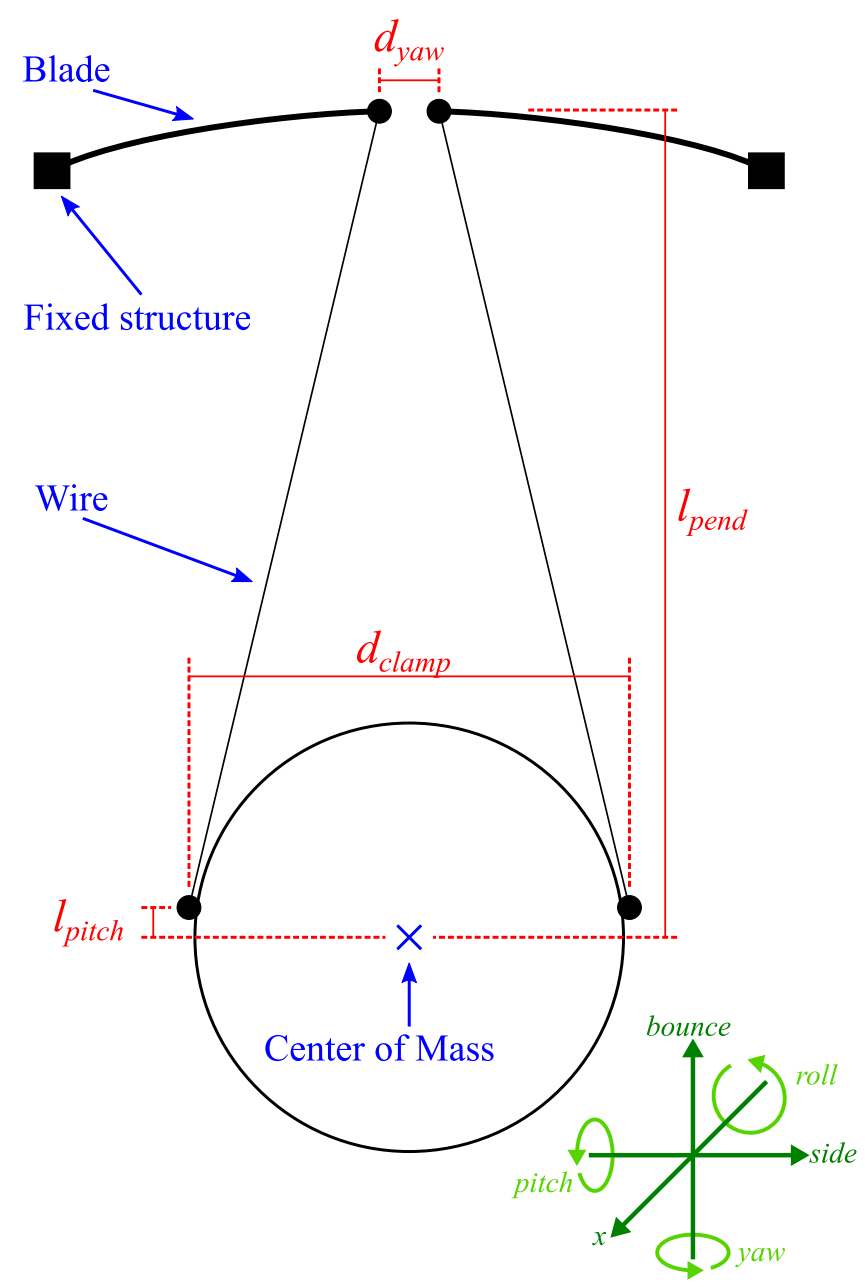

FIG. 2. A schematic representation of the HAUX suspension chain. The main functional elements are noted in blue. The six main degrees of freedom of the optic are identified in green in the bottom right corner. In red are shown the relevant dimensions that set the resonant frequency for $x$, pitch, and yaw.

Considering the expected performance of the Advanced LIGO seismic isolation platform on which the HAUX are mounted,$^{10}$ and even accounting for a safety factor, the HAUX requirements can be easily fulfilled by providing a single stage of isolation for $x$, pitch, and yaw, as long as the resonance frequency in each degree of freedom is kept below a few $\mathrm{Hz}$. Figure 2 shows a schematic representation of the configuration chosen for the HAUX suspensions, together with the geometrical parameters relevant to their performance. The optic is suspended from two wires, one on either side, which come close together at their upper attachment points; to a first approximation, the resonant frequency of $x$ is controlled by the length of the suspension wires $\left(l_{\text {pend }}\right)$, that of yaw is controlled by the horizontal separations of the upper $\left(d_{\text {yaw }}\right)$ and lower $\left(d_{\text {clamp }}\right)$ wire attachment points, and that of pitch is controlled by the height of the lower attachment points above the optic's center of mass $\left(l_{\text {pitch }}\right)$.

Below the Advanced LIGO measurement band, from 0.1 to $10 \mathrm{~Hz}$, the overall motion of the optic in pitch and yaw must be limited to $1 \mu \mathrm{rad}$ RMS for the alignment sensing and control subsystem to be able to maintain the interferometer close to its ideal working point. This requires the amplitude of motion at the resonant frequencies to be limited, which is 
TABLE I. Mass and intrinsic moment of inertial of the suspended assembly (optics, optic holder, and attachments) derived from nominal design parameters and used to calculate the final resonant frequencies.

\begin{tabular}{lc}
\hline \multicolumn{1}{c}{ Parameter } & Value \\
\hline \hline Mass & $374 \mathrm{~g}$ \\
Moment of inertia (for pitch) & $2.12 \times 10^{-4} \mathrm{~kg} \mathrm{~m}^{2}$ \\
Moment of inertia (for yaw) & $2.68 \times 10^{-4} \mathrm{~kg} \mathrm{~m}^{2}$ \\
Moment of inertia (for roll) & $4.35 \times 10^{-4} \mathrm{~kg} \mathrm{~m}^{2}$ \\
\hline \hline
\end{tabular}

accomplished via active damping using a set of four sensoractuators described in Section III.

An additional requirement for the HAUX is that the remaining three degrees of freedom of the optic must each have at least a single stage of isolation from the motion of the table, with a resonant frequency below $10 \mathrm{~Hz}$. This requirement is met by hanging the two suspension wires from two blade springs: the common or differential motion of the blade's tip allows for the optic to rotate around the optical axis (roll), swing orthogonal to it (side), or move along the vertical axis (bounce). Again, adjusting the geometrical and mechanical parameters allows controlling the resonance of these three degrees of freedom.

It should appear clear that this geometry arrangement provides all the physical parameters needed to adjust the resonances to desired values; approximate formulae to estimate such values are reported in the Appendix. However calculating the final transfer functions is not straightforward; the intuitive picture of independent harmonic oscillators acting along the different degrees of freedom is useful to understand how the various resonant frequencies can be controlled but is not accurate; in reality, $x$ and pitch, as well as roll and side, are degrees of freedom of double oscillator systems, and each pair combines to form two normal modes. In addition, the longitudinal and bending stiffness of the wires has a nonnegligible effect on the resonances of some of the modes. ${ }^{11}$ To calculate the values of the geometrical parameters needed to obtain the desired resonant frequency for each mode, and the exact composition of the normal modes, we used a semianalytical model implemented as a Mathematica package, ${ }^{12}$ which accounts for all these effects. We also used the finite element analysis software COMSOL Multiphysics to model the blade springs and to obtain the desired vertical spring constant. For these calculations, the inertial properties of the complete suspended assembly, as described in Section III, were derived from nominal mechanical dimensions and material properties and are reported in Table I.

The final values of the parameters and the resulting resonant frequencies are reported in Tables II and III. Note that

TABLE II. Values of geometrical parameters used in the final design of the HAUX.

\begin{tabular}{lc}
\hline \hline Parameter & Design value $(\mathrm{mm})$ \\
\hline$d_{\text {yaw }}$ & 15.7 \\
$d_{\text {clamp }}$ & 100.3 \\
$l_{\text {pitch }}$ & 1.0 \\
$l_{\text {pend }}$ & 259.3 \\
\hline \hline
\end{tabular}

TABLE III. Modeled and measured resonant frequencies of the six degrees of freedom of the optic; modeled results are calculated using exact nominal values from Table II. Measured values reported here, which have a $1 \sigma$ confidence interval equal to the last significant digit, refer to the final prototype; production units exhibit very similar values. Except for yaw, the measured resonant frequencies are systematically lower than modeled; this discrepancy is further discussed in Section IV B.

\begin{tabular}{lcc}
\hline \hline Mode & Modeled $\mathrm{f}_{0}(\mathrm{~Hz})$ & Measured $\mathrm{f}_{0}(\mathrm{~Hz})$ \\
\hline x/pitch 1 & 0.98 & 0.95 \\
x/pitch 2 & 1.12 & 1.04 \\
Yaw & 0.76 & 0.80 \\
Bounce & 7.19 & 6.14 \\
Side/roll 1 & 1.00 & 1.00 \\
Side/roll 2 & 10.63 & 8.97 \\
\hline \hline
\end{tabular}

since precise matching of pre-determined resonance frequency values was not a requirement, no mechanism has been incorporated in the HAUX design to compensate for machining and assembly tolerances and to fine-tune the resonance frequency values.

\section{MECHANICAL DESIGN}

The final HAUX mechanical design, in addition to accommodating the suspension chain described in Sec. II, had to satisfy a number of functional demands:

- being vacuum compatible at the particularly stringent level required for Advanced LIGO, which imposes restrictions not only on the total outgassing but also on the molecular species being outgassed;

- being able to accommodate $75 \mathrm{~mm}$ diameter mirrors with a clear aperture of at least $10 \mathrm{~mm}$, for horizontal angles of incidence up to $55^{\circ}$;

- providing active control of the optics in $x$, pitch, and yaw and passive damping for all other degrees of freedom;

- being equipped with safety stoppers to protect the optic in case of unexpected shaking or a wire failure and to allow the optic to be clamped in place when needed;

- allowing for fine tuning the optic working position in pitch to within $1 \mathrm{mrad}$, so as to mechanically remove any large offset from the active control system;

- having the lowest structural resonance above about $150 \mathrm{~Hz}$, to avoid interfering with the seismic-isolation table's active control system;

- last but not least, being as compact and simple as possible.

Assembly and maintenance of the suspensions and suspended optics are made more convenient by installing the $75 \mathrm{~mm}$ diameter, $25 \mathrm{~mm}$ thick optic in a lightweight aluminum holder, shown in Fig. 3(a). In this way, attachment clamps and actuation magnets can be attached to the holder rather than directly glued to the optic, allowing for easy replacement of the mirror with minimal disassembly of the suspension; the metal holder also allows to realize eddy current damping by attaching the strong magnets to the suspension's structure rather than to the suspended optics, thus reducing the coupling with environmental magnetic fields. The optic is held in position 

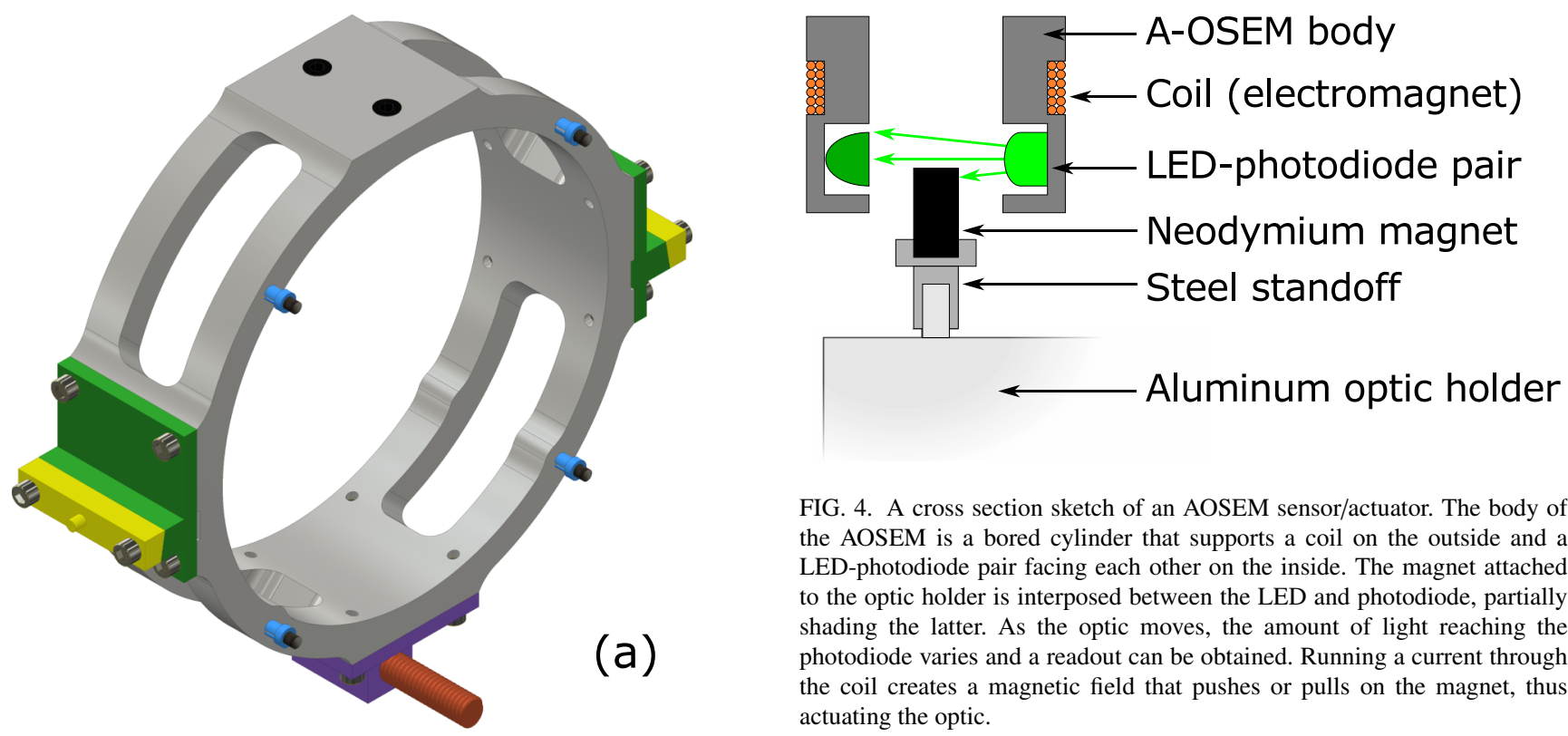

FIG. 4. A cross section sketch of an AOSEM sensor/actuator. The body of the AOSEM is a bored cylinder that supports a coil on the outside and a LED-photodiode pair facing each other on the inside. The magnet attached to the optic holder is interposed between the LED and photodiode, partially shading the latter. As the optic moves, the amount of light reaching the photodiode varies and a readout can be obtained. Running a current through the coil creates a magnetic field that pushes or pulls on the magnet, thus actuating the optic.

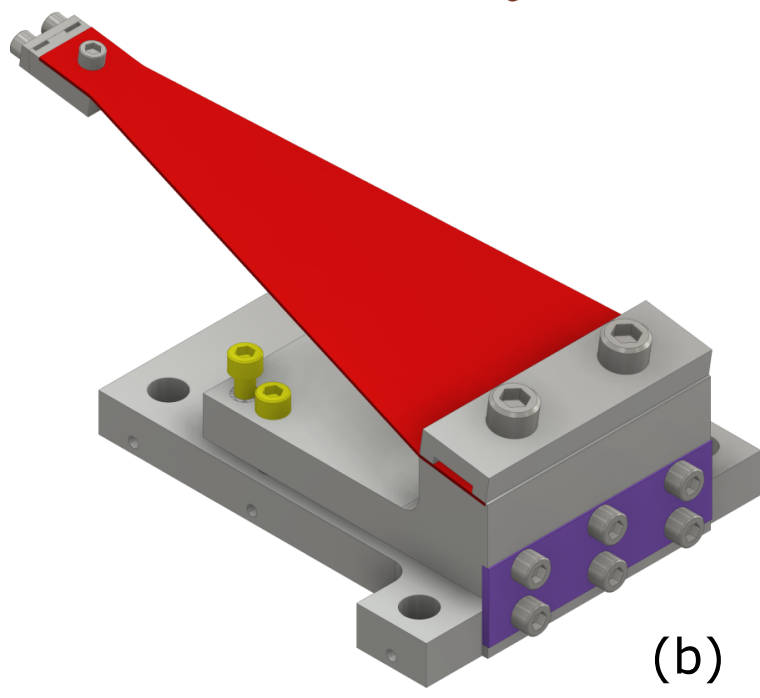

FIG. 3. (a) The aluminum optic holder used in the HAUX, seen from the back of the optic and represented in colors for clarity. Note the wire clamps (green and yellow) on the sides, the balancing rod (orange) at the bottom, and the magnets (black) together with their small steel standoffs (light blue). Also note the optic locking screws at the very top (black) and the four raised contact points at $\pm 45^{\circ}$ from the bottom, aligned with the position of the bottom magnets. (b) A blade spring assembly. The blade spring itself is represented in red; in purple is the steel flexure plate, and in yellow the push-pull screws.

by a pair of PEEK set screws that push it from the top against four raised contact points distributed on the front and back of the holder, at $\pm 45^{\circ}$ from the bottom. A wire clamp provided with an array of pre-machined vertical grooves is attached on either sides of the aluminum holder. Threading the suspension wire through one of the grooves helps defining its position into the clamp and avoids over squeezing, since the depth of the groove is dimensioned to optimally compress the wire when the clamp is completely closed; the array is necessary to be able to accommodate for the tolerance in the physical dimension of the different optics by selecting the groove whose horizontal position is closer to that of the center of mass of the as-built suspended assembly, thus avoiding big static pitch offsets. The optic holder also has a fine-threaded horizontal through hole at the bottom that allows for a copper rod to be screwed back and forth to move the center of mass of the assembly by small amounts and further adjust the static pitch of the optic.

Four magnets are magnetically attached to four steel sleeves, which are press-fitted over matching posts arranged in a $58.2 \mathrm{~mm}$ square pattern on the back of the holder. The magnets work together with the sensing/actuation units called AOSEMs. ${ }^{13}$ As shown in Fig. 4, an AOSEM is a combination of an electromagnetic actuator and a shadow sensor, in turn comprised of a light-emitting diode (LED) and a photodetector. The AOSEM is attached to the structure of the suspension in such a way that the magnet is coaxial with the coil and partially shields the photodetector from the light coming from the LED. As the magnet moves back and forth, the amount of light reaching the photodetector changes and a position readout can be obtained. At the same time, a current can be run through the coil, creating a magnetic field and a force on the magnet. By suitable linear combinations of the readout of the four AOSEMs, signals for $x$, pitch, and yaw can be obtained (three AOSEMs would be sufficient, but a fourth one has been introduced for redundancy, calibration, and symmetry purposes).

Two $250 \mathrm{~mm}$ long, $150 \mu \mathrm{m}$ diameter steel music wires run from the bottom clamps to smaller, single-groove clamps at the tips of the spring blades, shown in Fig. 3(b). The width profile of the monolithic, $0.5 \mathrm{~mm}$ thick stainless steel blades is comprised of three sections: a $9.5 \mathrm{~mm}$ wide, $9 \mathrm{~mm}$ long flat tip attached to the wire clamp, a $76.8 \mathrm{~mm}$ long tapered section that is free to flex, and a $40.6 \mathrm{~mm}$ wide, $12 \mathrm{~mm}$ long section clamped to an adjustable blade support. The variable width of the tapered section allows for the stress to be equally distributed along the blade when the tip is loaded. ${ }^{14}$ The support has a flexible joint and a system of push-pull screws that provide fine control of the departure angle of the blade, so that the working point of the tip can be made to be horizontal under load and for small angles, oscillate only in the vertical direction. According to an analytical study and a finite-element model, the resulting spring constant is $380 \mathrm{~N} \mathrm{~m}^{-1}$ and the maximum stress under 

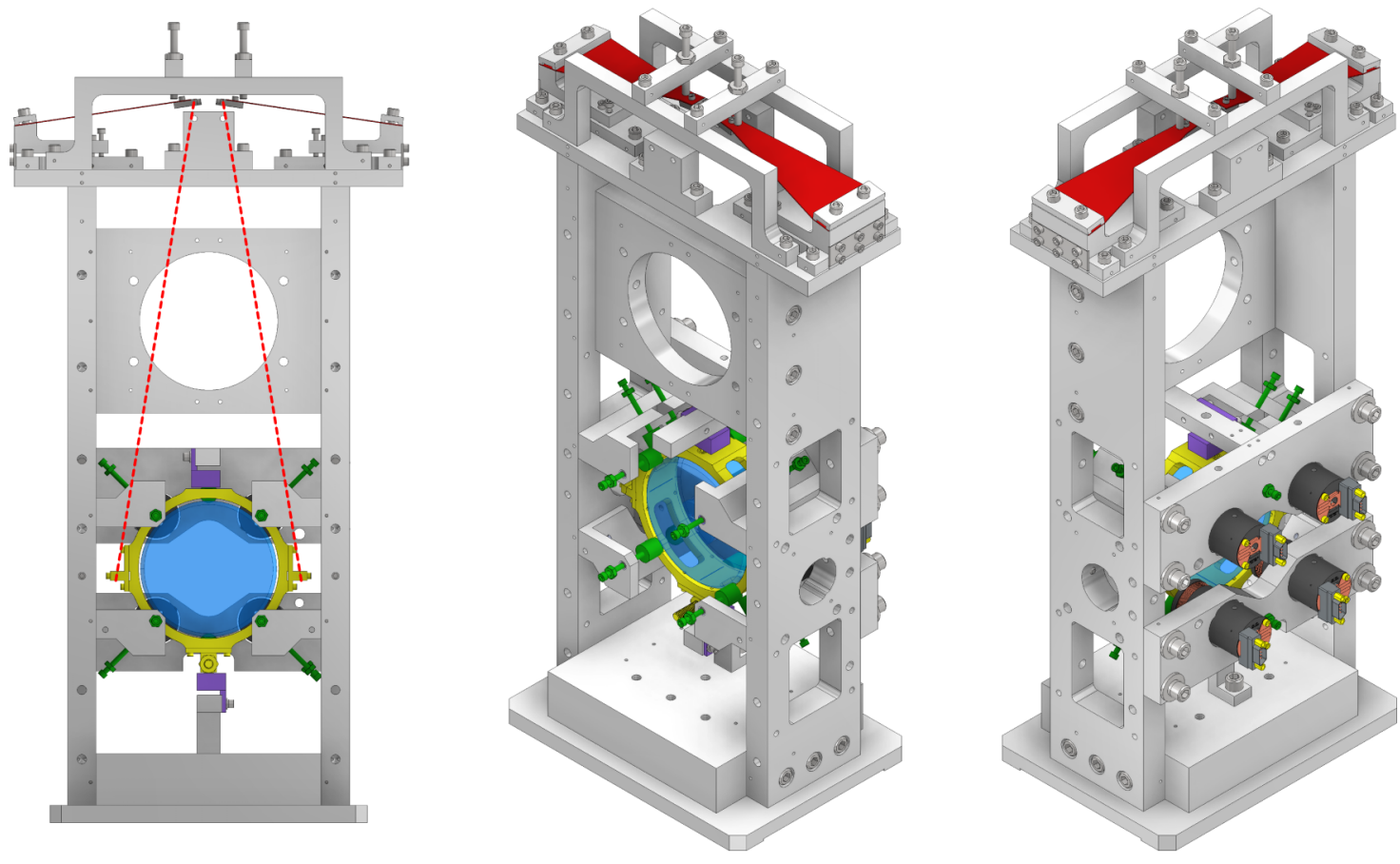

FIG. 5. Front and isometric views of a 3D model of the suspension, with relevant components highlighted in color: optic in blue; optic holder in yellow; locking screws in green; damping magnet casings in purple; AOSEMs in dark grey; blade springs in red. The wires are not shown in the 3D model, and the blades are represented unloaded (straight); the approximate placement of the wires is shown by the dashed red line in the front view.

load is approximately $85 \mathrm{MPa}$, less than $50 \%$ of the yield strength of stainless steel.

Figure 5 shows a 3D model of an assembled HAUX suspension. The main structure, made of aluminum to reduce weight, fits in an envelope of dimensions $127 \mathrm{~mm} \times 217 \mathrm{~mm}$ $\times 441 \mathrm{~mm}(D \times W \times H)$ and weighs approximately $6 \mathrm{~kg}$. It consists of a base, two side walls, two horizontal bars each supporting two AOSEMs, a stiffening slab connecting the two walls, and a top slab to which the blade supports are attached (using slotted holes to allow for precise adjustment during assembly). From the structures surrounding the optic, a set of 14 soft-tip screws protrude towards the aluminum holder and serve the purpose of safety stop and clamping devices in case of need. Two pairs of neodymium magnets are mounted in aluminum casings directly above and below the optic holder and provide passive damping via eddy currents. The magnets are anti-parallel, so as to minimize the field far from the suspension, and their distance from the holder can be adjusted from 0 to about $5 \mathrm{~mm}$ to obtain the desired level of damping. It should be noted that although the damping is intended to mainly affect the degrees of freedom not actively controlled by the AOSEMs, this arrangement of magnets has some damping effect also on $x$, pitch, and, much less, yaw.

\section{PERFORMANCE}

The final HAUX prototype, as well as the production units, has been tested for both compliance with the requirements and quality of manufacturing. ${ }^{15-17}$ Subsections IV A-IV D highlight the most important tests; when there are no significant differences between the various units, in the interest of clarity and space only a representative subset of results is presented.
Absolute calibration of most of these measurements involves independent calibration of a variety of software and hardware interfaces which are part of the Advanced LIGO control infrastructure, and not directly related to the HAUX design or their performance. Such calibration was often not available at the time the measurements were taken. For this reason, data are presented either in arbitrary units, or with a nominal calibration, depending on the case. Once the suspensions have been installed in the interferometer and the final components were available, the calibration of the entire chain, from software control interface to mirror response, has been verified to within $15 \%$ from the nominal value. ${ }^{18}$

\section{A. Pitch and yaw pointing range}

The mechanical limit to pitch and yaw rotation of the optic is set by the position of the safety stops, which can be adjusted to accommodate a range well beyond $10 \mathrm{mrad}$. The practical limit is then set by the available current through the coils, by the force per unit current that the AOSEMs can exert, $16 \times 10^{-3} \mathrm{~N} \mathrm{~A}^{-1}$ with the magnets in use, and by the rotational stiffness of pitch and yaw, which with the design values for $d_{\text {yaw }}, d_{\text {clamp }}$, and $l_{\text {pitch }}$ is of the order of $5 \times 10^{-3} \mathrm{Nm} / \mathrm{rad}$ for both degrees of freedom. The target dynamic range of $\pm 10 \mathrm{mrad}$ can thus be obtained with a current of $35 \mathrm{~mA}$, or the maximum range can be reduced improving noise and resolution, depending on the design of the current driver. The linearity of the actuation in the range of interest is also important. Figure 6 shows the rotation of the optic measured using an optical lever for one of the production units, for various values of the commanded actuation.

It is worth noting that the AOSEMs have a linear displacement readout range of about $\pm 350 \mu \mathrm{m}$ which, given 


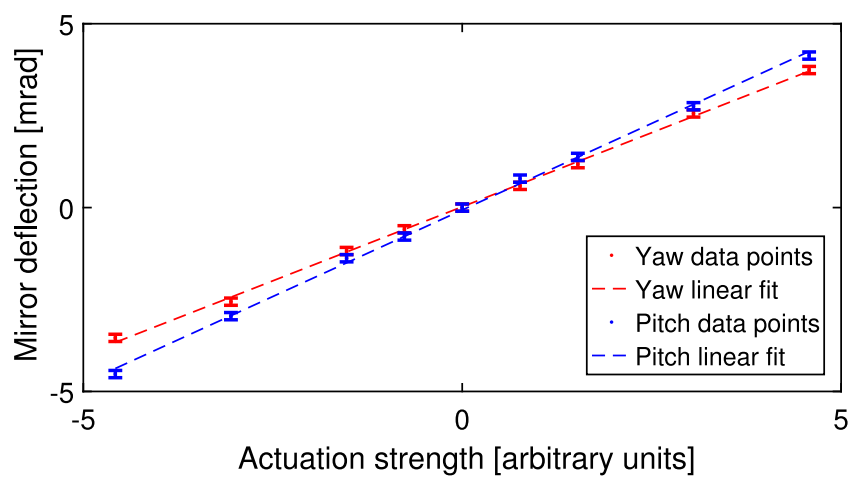

FIG. 6. Plot of the measured mirror deflection angle as a function of commanded actuation (arbitrary units). The response is linear over the entire actuation range of $\pm 5 \mathrm{mrad}$ ( $\pm 10 \mathrm{mrad}$ of beam deflection). The actuation is expressed in arbitrary units because calibration was not available for the preliminary electronic chain used at the time of this measurement.

the separation between the four units, results in a rotational readout range of about \pm 12 mrad for both pitch and yaw.

\section{B. Resonant frequencies and transfer functions}

The measured resonant frequencies for all six degrees of freedom are reported in Table III alongside the modeled values. These measurements were performed using the final prototype suspension built; however, the other eight production units assembled and installed in the Advanced LIGO detectors exhibit very similar values. It is apparent that there is a systematic tendency of the measured frequencies to be lower than the modeled ones. For the degrees of freedom dominated by the blade springs' motion this is expected, as the blades had been independently tested before installation and found to be softer than originally modeled (probably because of slightly different physical properties of the material used). For the other degrees of freedom, the origin of the discrepancy has not been identified. A study of the suspension computer model has shown that a number of realistic machining and assembly tolerances, as well as material property variations, can combine in several ways to explain the observed values. However, this has not been further investigated, since it was of no particular interest in the context of Advanced LIGO where lower resonant frequencies are actually an advantage.

With the exception of the decrease in the resonant frequencies discussed above, the measured transfer functions agree very well with the model. As an example, Fig. 7 shows a subset of transfer functions from force (or torque) to displacement (or rotation) of the optic for one particular production unit labeled H1-IM3. Again, all the assembled and tested units show comparable results, as exemplified in Fig. 8, which shows the force to motion in the $x$ degree of freedom transfer function, measured for 4 different units at the LIGO Hanford Observatory. Given that the HAUX do not incorporate any mechanism to fine-tune the resonances of the as-built units, the agreement is very satisfactory, and compatible with expected machining and assembly tolerances.

It should be pointed out that the main goal of the suspensions is that of isolating the motion of the optic from that of the suspension point, making the transfer functions shown here not
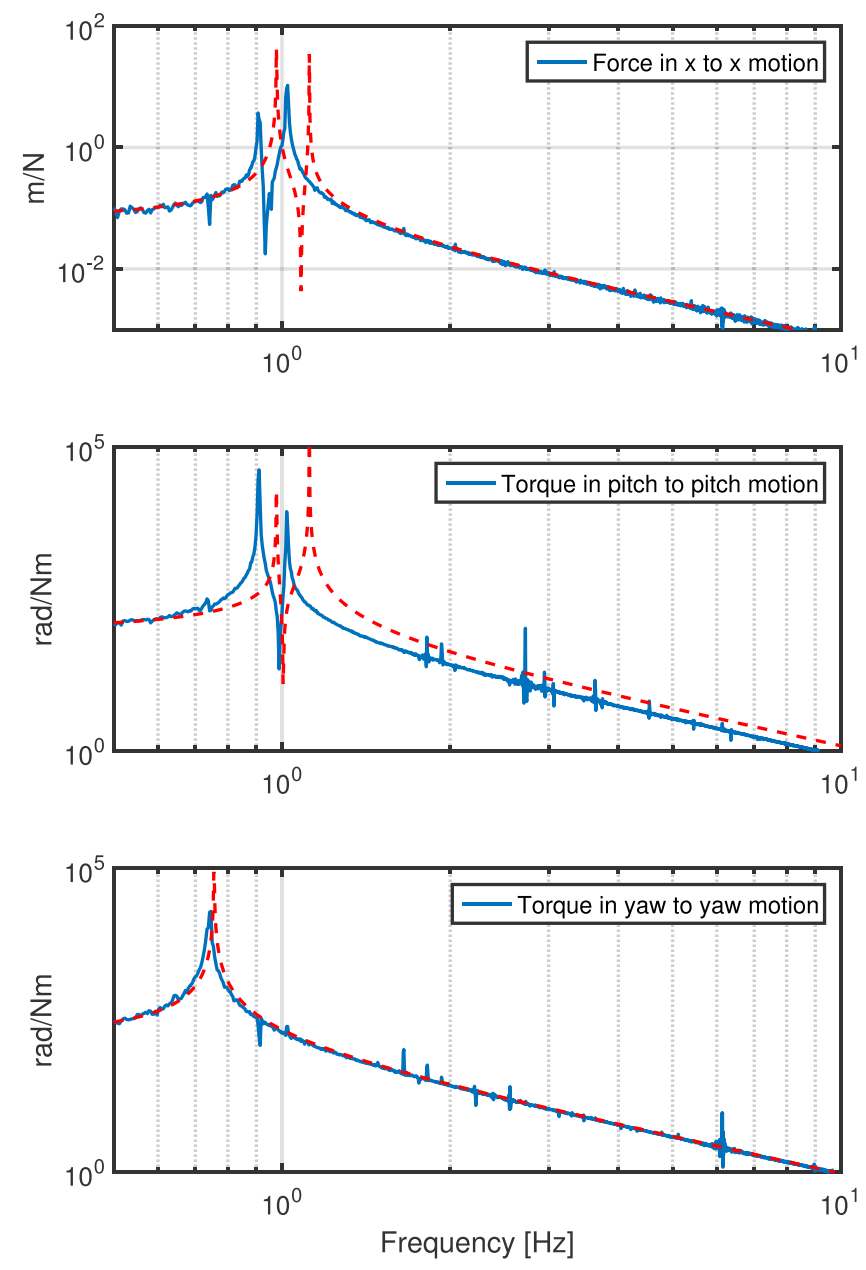

FIG. 7. Example transfer function from force (torque) to displacement (rotation) for one of the production units, LHO_IM3. Although the plots only show a frequency region around the main resonances for clarity of presentation, the transfer functions have been measured up to several hundred $\mathrm{Hz}$, where the test setup became limited by sensing noise; no notable deviations were observed form the expected $1 / f^{2}$ behavior already clearly visible in the above plots from about 2 to $10 \mathrm{~Hz}$.

be the ones we are most directly interested in. However, they can be measured more easily and accurately than transfer functions from motion of the suspension to motion of the optics, and being dependent on the same parameters, they provide an equally valid verification of the suspension performance and agreement with the model.

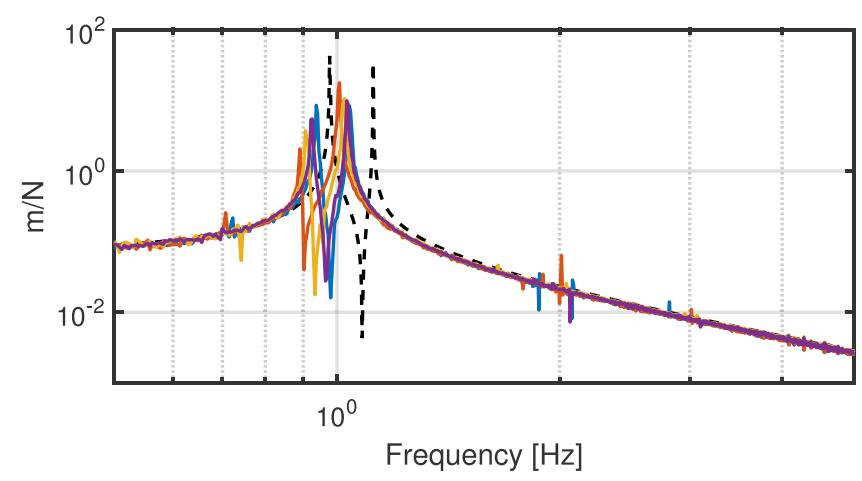

FIG. 8. Solid lines: transfer functions from force to motion in $x$, for the four different units installed at the LIGO Handford Observatory. Dashed line: model. 


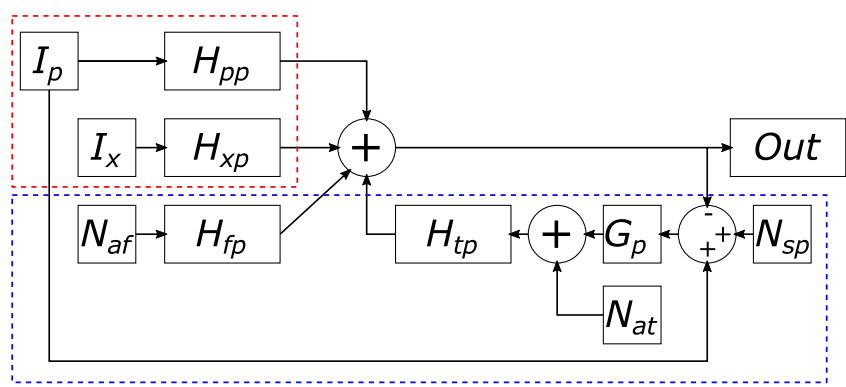

FIG. 9. A schematic representation of the model used to calculate the noise budget in pitch.

\section{Active and passive damping}

To estimate the noise performance and check compliance with the requirements during the design phase, we developed a noise model for each of the three actively controlled degrees of freedom (which are also the ones that most affect beam jitter and phase noise). As an example, Fig. 9 shows a schematic of the noise model developed for the pitch degree of freedom. The symbols are explained in Table IV.

The top left part of the diagram represents the direct effect of motion of the suspension structure, in both pitch and $x$, on optic pitch motion. The lower part represents the contribution of the AOSEMs: the differential pitch readout between optic and suspension structure, affected by sensing noise, is conditioned by the feedback loop gain to obtain the pitch torque actuation signal. This, together with torque and force actuation noise, affects the optic pitch through the relevant transfer functions. Although force and torque noise originating from the AOSEMs are not statistically independent, the correlation is small and we treated them as such for simplicity.

Solving the model for the noise in pitch yields

$$
\begin{aligned}
N_{\text {pitch }}= & \frac{1}{1+G_{p} H_{t p}}\left(I_{x} H_{x p}+N_{a f} H_{f p}+N_{a t} H_{t p}\right. \\
& \left.+N_{s p} G_{p} H_{t p}+I_{p}\left(H_{p p}+G_{p} H_{t p}\right)\right) .
\end{aligned}
$$

The various contributions to the final pitch noise, computed from input noise measured separately and transfer functions calculated from the Mathematica model, are plotted in Fig. 10. Here $G_{p}$ is a one-zero, two-poles bandpass filter selected for demonstration purposes; although not intended to be the final filter used in Advanced LIGO, it demonstrates that even this simple filter design is sufficient to meet requirements.

There are no specific requirements for the level of damping provided by the eddy current dampers; however, for non-

\begin{tabular}{|c|c|}
\hline Symbol & Explanation \\
\hline$H_{p p}, H_{x p}$ & $\begin{array}{l}\text { Transfer functions from } x \text { and pitch motion of the } \\
\text { platform, respectively, to pitch motion fo the optic }\end{array}$ \\
\hline$H_{f p}, H_{t p}$ & $\begin{array}{l}\text { Transfer functions from force and torque, } \\
\text { respectively, to pitch motion of the optic }\end{array}$ \\
\hline$I_{p}, I_{x}$ & Pitch and $x$ motion of the platform \\
\hline$N_{a f}, N_{a t}$ & Actuation force and torque noise, respectively \\
\hline$N_{s p}$ & Pitch sensing noise \\
\hline$G_{p}$ & Feedback loop gain \\
\hline
\end{tabular}

TABLE IV. Explanation of symbols used in Fig. 9 and Eq. (3).

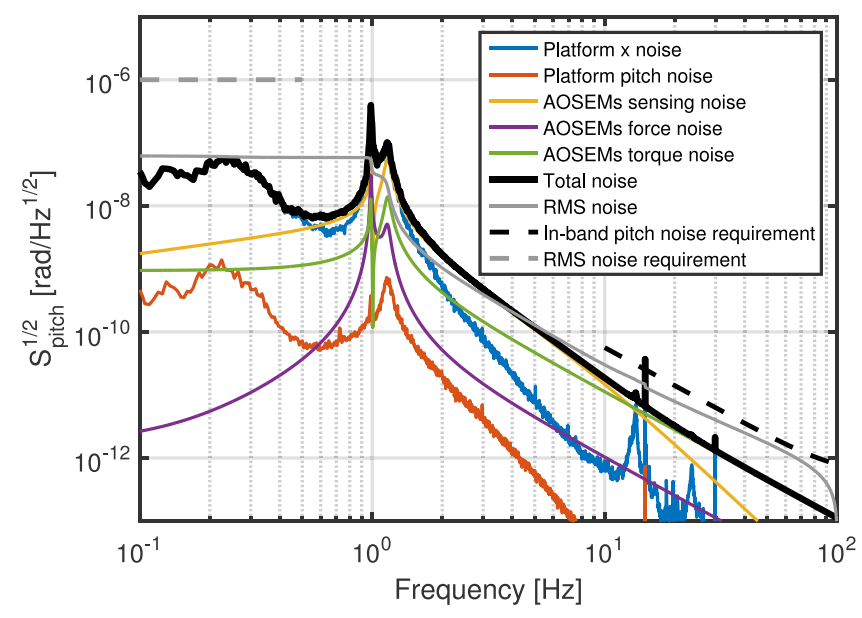

FIG. 10. The noise budget of the pitch degree of freedom based on the noise model represented in Fig. 9. The black and grey dashed lines represent the requirements on the total pitch noise and its RMS value, respectively, in the bands of interest. For this example, we set $G_{p}$ equal to a band-pass filter with a zero at $1 \mathrm{mHz}$ and two poles at $0.1 \mathrm{~Hz}$ and $1 \mathrm{~Hz}$; it can be seen that even this simple solution is sufficient to damp the resonances and keep the RMS noise within specifications, without exceeding the in-band noise requirement.

cavity suspensions in Advanced LIGO, it is generally considered adequate to obtain a quality factor, $Q$, somewhere between 10 and 100 to prevent the amplitude of motion of the uncontrolled degrees of freedom to grow too big. We designed the eddy current dampers to be adjustable, so that their effect could be varied over a wide range of values. Table $\mathrm{V}$ shows the reduction of $Q$ in the three relevant degrees of freedom, measured by observing the rate of decay of oscillations, for the nominal placement of the magnets, $2 \mathrm{~mm}$ away from the optic holder. As already mentioned at the end of Section III, the eddy current dampers also damp the actively controlled degrees of freedom, mostly $x$ and pitch. This is not a problem in Advanced LIGO because the resulting $Q$ is still higher than the value targeted by the active control.

\section{Structural resonances}

The mechanical resonances of the structure depend on the rigidity of the structure itself, and on to what and how the structure is clamped. Using a commercial system from Brüel \& Kjær, we have measured each set of HAUX structural resonances when they are installed and clamped in their final position on the Advanced LIGO optical tables. ${ }^{19,20}$ As an example, Fig. 11 shows the measurements taken for all 4 units installed at the Hanford Observatory. We found the measurements to be very consistent among different suspensions and to meet the requirement that the lower resonance be above $150 \mathrm{~Hz}$, with the only exception of a unit installed at the Livingston Observatory; this non-compliance, probably

TABLE V. Resonance quality factors measured with and without the eddy current dampers in their nominal position.

\begin{tabular}{lcc}
\hline \hline DoF & Q, undamped & Q, damped \\
\hline Side/roll 1 & $6000 \pm 1000$ & $74 \pm 2$ \\
Side/roll 2 & $500 \pm 25$ & $33 \pm 3$ \\
Bounce & $420 \pm 20$ & $43 \pm 3$ \\
\hline \hline
\end{tabular}



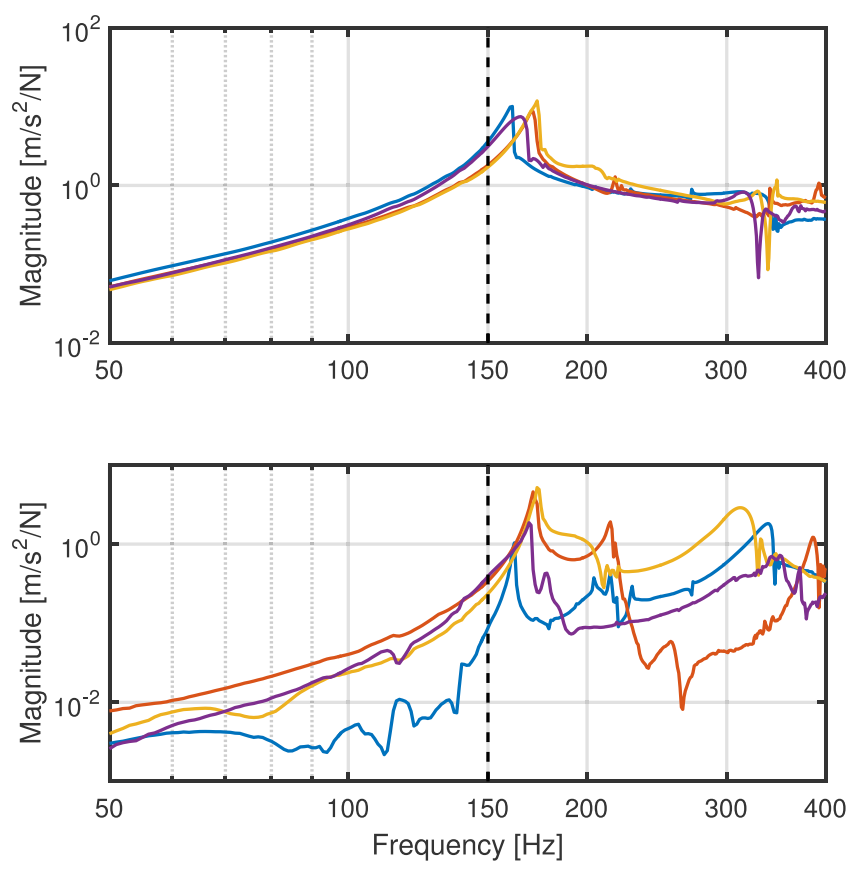

FIG. 11. Measurement of the mechanical resonances of the HAUX structure for all 4 units installed at the Hanford Observatory. A tri-axial accelerometer is attached to the structure, which is excited along the $x$ (top plot) and side (bottom plot) directions using a calibrated hammer. In each plot, the four traces represent the four units tested at LHO. Each trace is the root mean square of the accelerations measured by the accelerometer along each of the three axis, so as to highlight any resonant peak independently of the specific shape of the mechanical mode excited.

due to a manufacturing issue, does not appear to have any significant impact on the performance of the ISI platforms, but it is nevertheless scheduled to be further investigated when interferometer operations allow.

\section{CONCLUSIONS}

We have presented the design rationale and implementation of a compact single stage suspension for $75 \mathrm{~mm}$ diameter optics. The suspension provides isolation in all degrees of freedom, with resonant frequencies around $1 \mathrm{~Hz}$ for all but the bounce and roll modes, which are below $10 \mathrm{~Hz}$. The suspension has active control for the three most critical degrees of freedom of the optic and passive damping for the remaining three. The design incorporates a number of expedients to make installation/replacement and initial alignment of the optics more convenient. The suspension design meets the requirements for suspending out-of-cavity optics in the Advanced LIGO input optics chain and is versatile enough to be used in other precision optic experiments.

\section{ACKNOWLEDGMENTS}

The work presented in this paper has been supported by NSF Grant Nos. PHY-0855313 and PHY-0969935 and by a subcontract stipulated with the LIGO Laboratory.

\section{APPENDIX: ESTIMATE OF RESONANT FREQUENCIES}

In principle, the equations of motion of the suspended optic for small oscillations can be easily obtained in all degrees of freedom, for example, using Lagrangian mechanics. Once all couplings and wire bending effects are included, however, the expressions for the resonant frequencies become overly complex, requiring the use of a semi-analytical computer model to handle the exact solutions.

Here we provide basic estimates of the resonant frequencies for a generic suspension with a structure similar to the HAUX, and their dependence on the parameters listed in Fig. 2 and Table II; such estimates are sufficient for most applications, and even for the most demanding ones they can be a useful aid in the first stages of the design, before a full detailed model is developed. We consider small oscillations and expand at first order in the oscillation coordinate around the equilibrium point; further specific simplifying assumption is listed at the beginning of each subsection. In the following, $g$ indicates the gravitational acceleration, $m$ the mass, and $I_{\text {pitch }}, I_{\text {roll }}$, and $I_{\text {side }}$ the intrinsic (i.e., around the center of mass) moment of inertia of the suspended optic and holder assembly, relevant for the subscripted degree of freedom. Table VI compares the values obtained with these simple formulas with the results of the full semi-analytical model.

\section{1. $x$}

- The $x$ and pitch degrees of freedom do not mix (this is a reasonable assumption when $l_{\text {pend }} \gg l_{\text {pitch }}$ and $I_{\text {pitch }}$ $\gg m l_{\text {pitch }}^{2}$.

- Blades do not flex and wires are inextensible.

- Wires are infinitely flexible, i.e., the effective flexure point corresponds to the mechanical clamping point (break-off point).

We treat the $x$ motion of the optic as that of a point mass of a simple pendulum of length $l_{\text {pend }}$,

$$
f_{x} \approx \frac{1}{2 \pi} \sqrt{\frac{g}{l_{\text {pend }}}} .
$$

\section{Pitch}

- The $x$ and pitch degrees of freedom do not mix (this is a reasonable assumption when $l_{\text {pend }} \gg l_{\text {pitch }}$ and $I_{\text {pitch }}$ $\gg m l_{\text {pitch }}^{2}$.

TABLE VI. Estimation of resonant frequencies based on the parameters listed in Fig. 2 and Table II. The assumptions and formulas used for each degree of freedom are explained in the Appendix. For comparison, the last column lists the value corresponding to the closest normal mode computed with the full Mathematica model. The value in parenthesis for the estimated pitch resonance is obtained without applying the correction for $l_{\text {flex }}$ described in the text.

\begin{tabular}{lcc}
\hline \hline DoF & Basic model & Mathematica toolkit \\
\hline$x$ & 0.99 & 0.98 \\
Pitch & $0.97(0.59)$ & 1.12 \\
Yaw & 0.74 & 0.76 \\
Bounce & 7.10 & 7.19 \\
Roll & 10.24 & 10.63 \\
Side & 1.01 & 1.00 \\
\hline \hline
\end{tabular}


- Wires are infinitely flexible, i.e., the effective flexure point corresponds to the mechanical clamping point (break-off point).

To estimate the pitch motion of the optic, we assume that it behaves as a physical pendulum suspended by the lower attachment point of the wires,

$$
f_{\text {pitch }} \approx \frac{1}{2 \pi} \sqrt{\frac{m g l_{\text {pitch }}}{I_{\text {tot }}}}=\frac{1}{2 \pi} \sqrt{\frac{m g l_{\text {pitch }}}{I_{\text {pitch }}+m l_{\text {pitch }}^{2}}},
$$

where $I_{\text {tot }}$ is the total moment of inertia with respect to the pivoting point, equal to the sum of $I_{\text {pitch }}$ and $m l_{\text {pitch }}^{2}$, the moment of inertia of the center of mass with respect to the lower attachment point.

We note, however, that the assumption of infinitely flexible wires may not apply if $l_{\text {pitch }}$ is small. In the HAUX, the shift in the flexure point due to the wire's bending stiffness is of the same order of $l_{\text {ptich }}$, thus significantly increasing the resonant frequency. This can be accounted for by replacing $l_{\text {pitch }}$ with the sum $l_{\text {pitch }}+l_{\text {flex }}$ and estimating the flexure length as ${ }^{21}$

$$
l_{\text {flex }} \approx \sqrt{\frac{Y I}{T}},
$$

where $Y$ is the Young's modulus of the material, $T \approx \frac{m g}{2}$ is the tension, and $I=\frac{\pi}{4} r^{4}$ is the area's moment of inertial for a wire of circular cross section.

\section{Yaw}

- Blades do not flex and wires are inextensible.

- Wires are infinitely flexible, i.e., the effective flexure point corresponds to the mechanical clamping point (break-off point) and they have no torsional stiffness.

We estimate the resonant frequency in yaw by calculating the change in the height of the center of mass upon rotation of the optic and the associated increase in potential energy $\Delta U$. At second order in the rotation angle $\theta$, this is equal to

$$
\Delta U \approx \frac{m g}{2\left(l_{\text {pend }}-l_{\text {pitch }}\right)} \frac{d_{\text {yaw }}}{2} \frac{d_{\text {clamp }}}{2} \theta^{2}
$$

which results in a resonant frequency

$$
f_{\text {yaw }} \approx \frac{1}{2 \pi} \sqrt{\frac{m g d_{\text {yaw }} d_{\text {clamp }}}{4\left(l_{\text {pend }}-l_{\text {pitch }}\right) I_{\text {yaw }}}} .
$$

\section{Bounce}

- Each blade spring acts in the direction of the wire attached to it, and we treat blade and wire as two springs in series.

- We ignore the change in the angle of the wires upon vertical displacement of the optics.

The bounce motion is simply that of a mass-spring system with the elastic constant given by the combination of the blades and wires,

$$
k_{\text {eff }} \approx\left(\frac{1}{k_{\text {blade }}}+\frac{1}{k_{\text {wire }}}\right)^{-1}
$$

and

$$
f_{\text {bounce }} \approx \frac{1}{2 \pi} \sqrt{\frac{2 k_{e f f}}{m}} .
$$

\section{Roll}

- The roll and side degrees of freedom do not mix.

- Each blade spring acts in the direction of the wire attached to it, and we treat blade and wire as two springs in series.

We estimate the restoring torque for the roll mode by calculating the change in length of the effective spring composed of a wire and blade, upon rotation of the optic by an angle $\theta$ around the center of mass; we also assume that the corresponding elastic force acts in the direction of the wire at equilibrium. For a single effective spring the torque is

$$
N \approx k_{\text {eff }} \frac{\left(d_{\text {clamp }} l_{\text {pend }}-d_{\text {yaw }} l_{\text {pitch }}\right)^{2}}{\left(d_{\text {yaw }}-d_{\text {clamp }}\right)^{2}+4\left(l_{\text {pend }}-l_{\text {pitch }}\right)^{2}} \theta .
$$

While one spring is stretched, the other is shortened by the same length, and both contribute the same torque (note that this is true as long as the wire tension never drops to zero, which is true for small enough oscillations). The resonant frequency is then

$f_{\text {bounce }} \approx \frac{1}{2 \pi} \sqrt{\frac{2 k_{\text {eff }}\left(d_{\text {clamp }} l_{\text {pend }}-d_{\text {yaw }} l_{\text {pitch }}\right)^{2}}{\left(d_{\text {yaw }}-d_{\text {clamp }}\right)^{2}+4\left(l_{\text {pend }}-l_{\text {pitch }}\right)^{2}} \frac{1}{I_{\text {roll }}}}$.

\section{Side}

- The roll and side degrees of freedom do not mix.

- Blades do not flex and wires are inextensible.

- Wires are infinitely flexible, i.e., the effective flexure point corresponds to the mechanical clamping point (break-off point).

We calculate the increase in potential energy upon a lateral displacement $y$ of the optic, assuming inextensible wires, and expand to second order in $y$

$\Delta U \approx m g \frac{\left(d_{\text {clamp }}-d_{\text {yaw }}\right)^{2}+4\left(l_{\text {pend }}-l_{\text {pitch }}\right)^{2}}{8\left(l_{\text {pend }}-l_{\text {pitch }}\right)^{3}} y^{2}$

and the resulting frequency

$$
f_{\text {side }} \approx \frac{1}{2 \pi} \sqrt{\frac{g\left(\left(d_{\text {clamp }}-d_{\text {yaw }}\right)^{2}+4\left(l_{\text {pend }}-l_{\text {pitch }}\right)^{2}\right)}{4\left(l_{\text {pend }}-l_{\text {pitch }}\right)^{3}}} .
$$

${ }^{1}$ B. P. Abbott, R. Abbott, T. D. Abbott, M. R. Abernathy, F. Acernese, K. Ackley, C. Adams, T. Adams, P. Addesso, R. X. Adhikari et al., (LIGO Virgo Scientific Collaboration), Phys. Rev. Lett. 116, 061102 (2016).

${ }^{2}$ D. Martynov, E. Hall, B. Abbott, R. Abbott, T. Abbott, C. Adams, R. Adhikari, R. Anderson, S. Anderson, K. Arai et al., (LIGO Virgo Scientific Collaboration), Phys. Rev. D 93, 112004 (2016).

${ }^{3}$ C. L. Mueller, M. A. Arain, G. Ciani, R. T. DeRosa, A. Effler, D. Feldbaum, V. V. Frolov, P. Fulda, J. Gleason, M. Heintze, K. Kawabe, E. J. King, K. Kokeyama, W. Z. Korth, R. M. Martin, A. Mullavey, J. Peold, V. Quetschke, D. H. Reitze, D. B. Tanner, C. Vorvick, L. F. Williams, and G. Mueller, Rev. Sci. Instrum. 87, 014502 (2016).

${ }^{4}$ B. J. J. Slagmolen, A. J. Mullavey, J. Miller, D. E. McClelland, and P. Fritschel, Rev. Sci. Instrum. 82, 125108 (2011) 
${ }^{5}$ J. Hazel and S. Kawamura, "Small optics suspension (SOS) final design (mechanical system)," Technical Report LIGO-T970135, LIGO Document Control Center,1997.

${ }^{6}$ M. Fine, J. Hazel, J. Heefner, S. Kawamura, and S. Miyoki, "Small optics suspension (SOS) prototype test results," Technical Report LIGO-T960179, LIGO Document Control Center, 1997.

${ }^{7}$ The LIGO Document Control Center (DCC) is an online repository of documents produced by members of LIGO and the LIGO Scientific Collaboration. It is accessible from any modern web browser at the url https://dcc.ligo.org.

${ }^{8}$ G. Ciani, M. Arain, S. Aston, D. Feldbaum, P. Fulda, J. Gleason, M. Heintze, R. Martin, C. Mueller, D. M. Nanda Kumar, A. Pele, D. Reitze, P. Sainathan, D. Tanner, L. Williams, and G. Mueller, "HAM auxiliary suspensions design and testing documentation," Technical Report LIGOE1600169, LIGO Document Control Center, 2016.

${ }^{9}$ G. Mueller, Opt. Express 13, 7118 (2005).

${ }^{10}$ F. Matichard, B. Lantz, R. Mittleman, K. Mason, J. Kissel, B. Abbott, S. Biscans, J. McIver, R. Abbott, S. Abbott, E. Allwine, S. Barnum, J. Birch, C. Celerier, D. Clark, D. Coyne, D. DeBra, R. DeRosa, M. Evans, S. Foley, P. Fritschel, J. A. Giaime, C. Gray, G. Grabeel, J. Hanson, C. Hardham, M. Hillard, W. Hua, C. Kucharczyk, M. Landry, A. L. Roux, V. Lhuillier, D. Macleod, M. Macinnis, R. Mitchell, B. O'Reilly, D. Ottaway, H. Paris, A. Pele, M. Puma, H. Radkins, C. Ramet, M. Robinson, L. Ruet, P. Sarin, D. Shoemaker, A. Stein, J. Thomas, M. Vargas, K. Venkateswara, J. Warner, and S. Wen, Classical Quantum Gravity 32, 185003 (2015).
${ }^{11}$ C. I. E. Torrie, "Development of suspensions for the GEO 600 gravitational wave detector," Ph.D. thesis, University of Glasgow, 2000 http://www2. mpq.mpg.de/ ros/geo600_docu/text/theses/Calum_Torrie.pdf.

${ }^{12}$ M. Barton, Mathematica Pendulum Modeling Toolkit, 2016, https://labcit. ligo.caltech.edu/ e2e/SUSmodels/.

${ }^{13}$ L. Carbone, S. M. Aston, R. M. Cutler, A. Freise, J. Greenhalgh, J. Heefner, D. Hoyland, N. A. Lockerbie, D. Lodhia, N. A. Robertson, C. C. Speake, K. A. Strain, and A. Vecchio, Classical Quantum Gravity 29, 115005 (2012).

${ }^{14} \mathrm{~N}$. A. Robertson, "Design notes for production blades for HAM suspensions," Technical Report LIGO-T1000351, LIGO Document Control Center, 2010

${ }^{15} \mathrm{G}$. Ciani, "HAM auxiliary suspensions modeling and test results," Technical Report LIGO-T1000339, LIGO Document Control Center, 2011.

${ }^{16}$ G. Ciani, "ALIGO IO L1 HAM AUX test results," Technical Report LIGOT1300396, LIGO Document Control Center, 2013.

${ }^{17}$ G. Ciani, "ALIGO IO H1 HAM AUX test results," Technical Report LIGOT1300397, LIGO Document Control Center, 2013.

${ }^{18}$ D. Feldbaum, LIGO Livingston electronic logbook, entry 4689, 2012, https://alog.ligo-la.caltech.edu/aLOG/index.php?callRep=4689.

${ }^{19}$ A. P. Jeff Kissel, LIGO Hanford Electronic Logbook, Entry 5652, 2013, https://alog.ligo-wa.caltech.edu/aLOG/index.php?callRep=5652.

${ }^{20}$ P. F. Stuart Aston, LIGO Livingston Electronic Logbook, Entry 3948, 2012, https://alog.ligo-la.caltech.edu/aLOG/index.php?callRep=3948.

${ }^{21}$ G. Cagnoli, J. Hough, D. DeBra, M. Fejer, E. Gustafson, S. Rowan, and V. Mitrofanov, Phys. Lett. A 272, 39 (2000). 